PoS $\quad \begin{aligned} & \text { PROCEEDINGS } \\ & \text { OF SCIENCE }\end{aligned}$

\title{
Measurement of the forward-backward charge asymmetry in top quark production in proton-antiproton collisions at $1.96 \mathrm{TeV}$
}

Regina Demina*

University of Rochester

E-mail: deminaefnal.gov

We report on the D0 measurement of the top-antitop charge production asymmetry based on $5.4 \mathrm{fb}^{-1}$ of data. Asymmetry dependence on the invariant mass of the top-antitop system, its transverse momentum, as well as the number of extra jets is discussed.

The 2011 Europhysics Conference on High Energy Physics-HEP 2011,

July 21-27, 2011

Grenoble, Rhône-Alpes France

${ }^{*}$ Speaker. 
Over the last five years both Tevatron experiments have been consistently reporting positive forward-backward asymmetries in the production of top-antitop pairs $[1,2,3]$. The reported values are above the Standard Model prediction [4] with growing significance. In these proceedings we report on the new result from the D0 experiment based on $5.4 \mathrm{fb}^{-1}$ of data.

We define the forward(backward) direction to be that of the incoming proton(antiproton). In proton-antiproton collisions top-antitop pair are predominantly produced via quark-antiquark annihilation. Since energy required to produce a heavy top-antitop pair is fairly large it is mostly valence quarks that participate in this process. As a result the direction of the proton(antiproton) coincides with that of the incoming quark(antiquark). Thus, positive asymmetry means that top(antitop) quarks are produced preferentially in the direction of incoming quarks(antiquarks). Forwardbackward asymmetry in top-antitop pair production is defined through the difference in rapidity of top and antitop quarks:

$$
\begin{gathered}
\Delta y=y_{t}-y_{\bar{t}}, \\
A_{\mathrm{FB}}=\frac{N_{\mathrm{F}}-N_{\mathrm{B}}}{N_{\mathrm{F}}+N_{\mathrm{B}}},
\end{gathered}
$$

where $N_{\mathrm{F}}$ is the number of events that have $\Delta y>0$, and $N_{\mathrm{B}}$ is the number of events with $\Delta y<0$. Rapidities of top and antitop can be defined at production or after the full event selection and reconstruction. The corresponding asymmetries are then determined at the production or reconstruction levels. In previously reported results D0 quoted only reconstruction level asymmetry [1], while CDF performed unfolding to extract the production level asymmetry [3]. In the analysis reported in this conference, D0 presented unfolded results as well, which allows for a directed comparison between the two experiments. The detailed description of the D0 analysis is submitted for publication [5]. In these proceedings we only briefly outline the analysis and concentrate on the interpretation of the result within the Standard Model.

In the Standard Model top quark is expected to decay almost exclusively to b-quark and Wboson. Thus, top samples are labeled by the decay of W-bosons. We reconstruct top-antitop pair in the lepton+jets sample, where one W-boson decayed leptonically and the other hardonically. We require exactly one isolated lepton (electron or muon), missing energy due to an undetected neutrino and at least four jets. Transverse momenta of all objects must be above $20 \mathrm{GeV}$. We require that at least one jet is tagged as associated with a b-quark. The assignment of objects to top or antitop decay is done using a kinematic reconstruction, where the decay products of both W-bosons (two non-b jets or a lepton and a neutrino) are constrained to have an invariant mass of a W-boson, and decay products of both top and antitop quarks have an invariant mass of 172.5 $\mathrm{GeV}$. Only the solution with the lowest $\chi^{2}$ is kept for further analysis. Based on the rapidity difference between the reconstructed top and antitop (Fig. 1) the asymmetry is measured to be $A_{\mathrm{FB}}=(9.2 \pm 3.7) \%$. For comparison with the Standard Model prediction we chose to use the nextto-leading order event generator MC@NLO [6] interfaced with HERWIG [7] to perform parton showering. Simulated events are then processed by the full detector simulation. The predicted asymmetry at the reconstruction level is $(2.4 \pm 0.7) \%$. CDF reported strong dependence of the asymmetry on the invariant mass of the top-antitop system. D0 checked for this behavior. The observed asymmetry is consistent with observations by CDF, but the dependence is not nearly as strong (Fig. 2). 


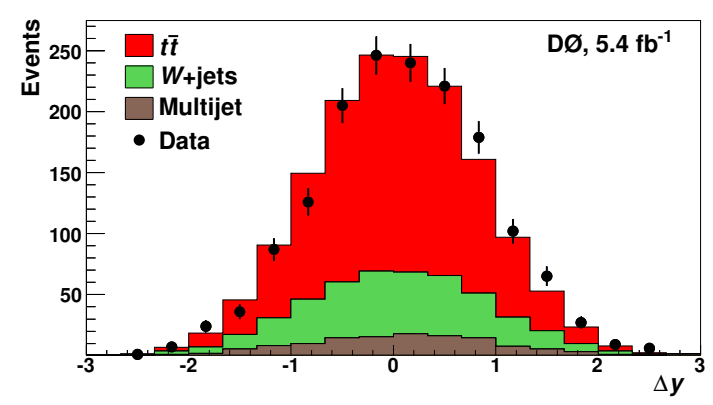

Figure 1: The reconstructed $\Delta y$. Bin widths correspond to about half of the detector resolution in $\Delta y$.

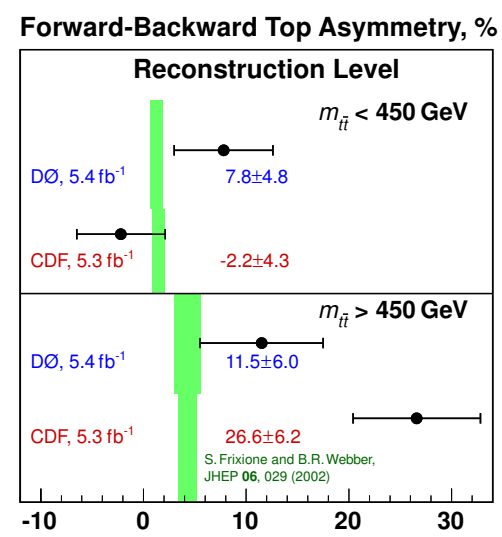

Figure 2: $A_{\mathrm{FB}}$ dependence on the invariant mass of the top-antitop system. D0 and CDF results are compared to the MC@NLO prediction (green band).

The selection and reconstruction of the top and antitop quarks smear their direction and thus dilute the true asymmetry. To infer the asymmetry at the production we employ fine bin unfolding with regularization. We take into the account the effects of the acceptance on the $\Delta y$ distribution as well as the probability for events to migrate from one bin to another. Since for the definition of the asymmetry it is most critical to evaluate the migration across $\Delta y=0$ boundary the distribution is binned using width of smaller bin size in this region. Typically, when bins size is reduced it leads to large statistical fluctuations in this region. We employ regularization technique to deal with this problem. The performance of this procedure is calibrated using ensembles of pseudo data sets. The resultant asymmetry at the production level is $(19.6 \pm 6.5) \%$ to be compared with the MC@NLO prediction of $(5.0 \pm 0.1) \%$.

In addition to fully reconstructing the top-antitop system we define asymmetry based on the rapidity of the lepton multiplied by its $\operatorname{sign}\left(q_{l} y_{l}\right)$ :

$$
A_{\mathrm{FB}}^{l}=\frac{N_{\mathrm{F}}^{l}-N_{\mathrm{B}}^{l}}{N_{\mathrm{F}}^{l}+N_{\mathrm{B}}^{l}}
$$

where $N_{\mathrm{F}}^{l}$ is the number of events that have $q_{l} y_{l}>0$, and $N_{\mathrm{B}}^{l}$ is the number of events with $q_{l} y_{l}<0$. The distribution in the signed lepton rapidity is presented in Fig. 3 and the corresponding asymmetry is $A_{\mathrm{FB}}^{l}=(14.2 \pm 3.8) \%$ at the reconstruction level and $A_{\mathrm{FB}}^{l}=(15.2 \pm 4.0) \%$ at the production 
level. MC@NLO-based expectation for this quantity is $(2.1 \pm 0.1) \%$.

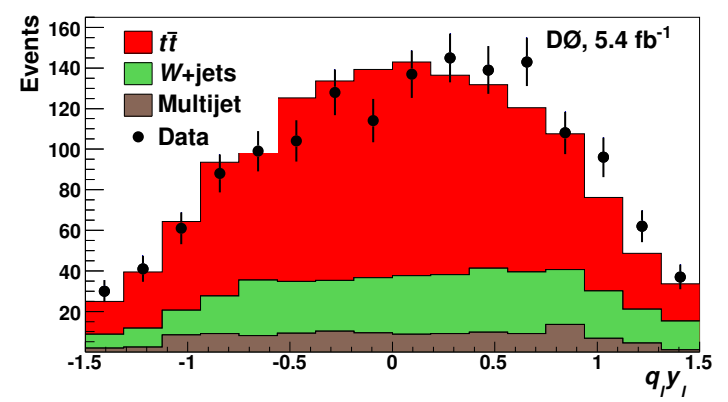

Figure 3: The reconstructed charge-signed lepton rapidity.

To summarize the measurements, both CDF and D0 report asymmetries that exceed the MC@ NLO prediction by more than two standard deviations. A number of publications seek to explain this effect by employing different beyond the Standard Model scenarios [8]. It is worth noting that most of these models come into some level of contradiction with data in other processes. It seems appropriate to explore a possibility of explaining this effect within the Standard Model. Within QCD the asymmetry in top-antitop production arises in the $\alpha_{s}^{3}$ order. Full $\alpha_{s}^{4}$ calculation for top-antitop production does not exist yet. Interference between tree level production and a box-diagram results in a two-body production, while the production of top and antitop in association with an extra gluon is a result of interference of processes involving initial and final state radiation. While in the former case the transverse momentum of the top-antitop system is expected to be very small and no extra objects are expected to be produced, in the later case the extra gluon may or may not result in an extra jet and the system will have a sizable $p_{T}$. D0 compared the reconstruction level asymmetry for events with an extra jet above $20 \mathrm{GeV}$ and for events without any extra jets to the MC@NLO prediction (see Table 1).

\begin{tabular}{|l|c|c|}
\hline & lepton +4 jets & lepton $+\geq 5$ jets \\
\hline Data $A_{\mathrm{FB}}(\%)$ & $12.2 \pm 4.2$ & $-3.0 \pm 7.8$ \\
$\mathrm{MC} @ \mathrm{NLO} A_{\mathrm{FB}}(\%)$ & $3.9 \pm 0.3$ & $-2.9 \pm 0.7$ \\
\hline
\end{tabular}

Table 1: Asymmetry measured in the sample with exactly 4 jets and with 5 or more jets.

In its analysis D0 also explores other observables that may help to shed some light on the origin of the asymmetry, in particular the transverse momentum of the top-antitop system, $p_{T}^{t \bar{t}}$. We found that MC@NLO interfaced with HERWIG predicts a strong dependence of the asymmetry on the $p_{T}^{t \bar{t}}$. To better understand the origin of this effect we ran PYTHIA ${ }^{1}$ using different tunes [11] and found that the effect is present when the angular coherence of the extra radiation is forced and absent otherwise (see Fig. 4). We observed that D0 data exhibits a softer $p_{T}^{t \bar{t}}$ spectrum than predicted by MC@NLO. To test this hypothesis we ran PYTHIA with initial state radiation turned completely off and found a better agreement with the data (see Fig. 5). In combination with the asymmetry

\footnotetext{
${ }^{1}$ As a tree level generator PYTHIA can not predict the overall asymmetry, yet it can be used to qualitatively study the dependence of the asymmetry on the kinematic properties of the event.
} 


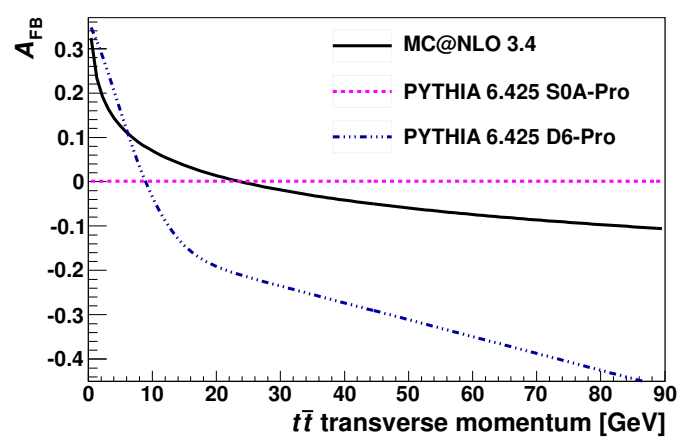

Figure 4: The $t \bar{t}$ asymmetry versus $p_{T}^{t \bar{t}}$ as predicted by MC@ NLO+HERWIG. For comparison, the predictions from PYTHIA with different tunes are also shown.
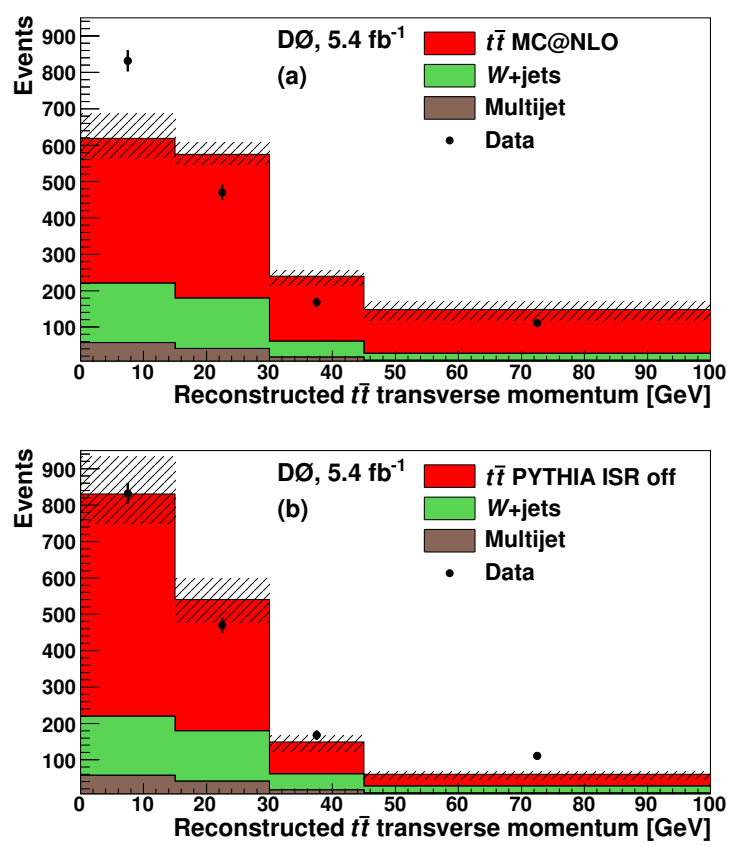

Figure 5: Reconstructed top pair transverse momentum for selected events. The dashed band represents systematic uncertainties due to the jet energy scale and resolution. Data points are compared to predictions based on MC@NLO (a) and PYTHIA with ISR off (b). Bin width is equal to half of the detector resolution in $p_{T}^{t \bar{t}}$.

dependence on $p_{T}^{t \bar{t}}$ softer spectrum would favor a larger positive asymmetry than predicted by MC@NLO in qualitative agreement with the Tevatron results. Recent calculations that include the contributions from electro-weak processes suggest an increased positive asymmetry [9]. LHC experiments report smaller asymmetry in top-antitop pair production [10], yet these results do not contradict the Tevatron measurement, since in proton-proton collisions the production is dominated by intrinsically symmetric gluon-gluon fusion.

In conclusion, we note that analysis of the full data set collected by both Tevatron experiments, 
as well as full $\alpha_{s}^{4}$ theoretical calculation would help to decide if there is any tension between the Standard Model and the experimental observations in the asymmetry of top-antitop pair production.

\section{References}

[1] V. M. Abazov et al. (D0 Collaboration), Phys. Rev. Lett. 100, 142002 (2008).

[2] T. Aaltonen et al. (CDF Collaboration), Phys. Rev. Lett. 101, 202001 (2008).

[3] T. Aaltonen et al. (CDF Collaboration), Phys. Rev. D 83, 112003 (2011).

[4] L. G. Almeida, G. Sterman, and W. Vogelsang, Phys. Rev. D 78, 014008 (2008); M. T. Bowen, S. D. Ellis, and D. Rainwater, Phys. Rev. D 73, 014008 (2006); S. Dittmaier, P. Uwer, and S. Weinzierl, Phys. Rev. Lett. 98, 262002 (2007); K. Melnikov and M. Schulze, Nucl. Phys. B 840, 129 (2010); J. H. Kuhn and G. Rodrigo, arXiv:1109.6830 [hep-ph] (2011).

[5] V. M. Abazov et al. (D0 Collaboration), arXiv:1107.4995 [hep-ph] (2011), submitted to Phys. Rev. D.

[6] S. Frixione and B. R. Webber, J. High Energy Phys. 06, 029 (2002);

S. Frixione et al., J. High Energy Phys. 08, 007 (2003).

[7] G. Corcella et al., J. High Energy Phys. 01, 010 (2001).

[8] S. Westhoff, these proceedings: arXiv:1108.3341[hep-ph] (2011).

[9] W. Hollik and D. Pagani, arXiv:1107.2606 [hep-ph] (2011).

[10] CMS Collaboration, CMS-PAS-TOP-11-014, 2011; ATLAS Collaboration, ATLAS-CONF-2011-106.

[11] P. Z. Skands, Phys. Rev. D 82, 074018 (2010). 